\title{
Colitis as a Sole Presentation of SARS-CoV-2 Infection: Case Report
}

\author{
Siraya Jaijakul ${ }^{1}$ \\ Accepted: 2 June 2020 / Published online: 11 June 2020 \\ (C) Springer Nature Switzerland AG 2020
}

\begin{abstract}
On March 11, 2020, COVID-19 was declared as a pandemic by World Health Organization. Criteria for identifying persons under investigation for SARS-CoV-2 infection by Centers for Disease Control and Prevention remain focusing on fever and respiratory symptoms. We report a case of COVID-19 patient who presented with colitis alone.
\end{abstract}

Keywords COVID-19 · SARS-CoV-2 infection · Colitis

\section{Introduction}

Coronavirus Disease 2019 (COVID-19), caused by severe acute respiratory syndrome coronavirus 2 (SARS-CoV-2), has emerged in Wuhan city, China since December 2019. COVID-19 has rapidly spread worldwide and was declared as a pandemic by World Health Organization on March 11, 2020 [1]. Persons with underlying diabetes mellitus, chronic lung disease, and cardiovascular disease appear to be at higher risk for severe COVID-19-associated disease [2]. Patients with COVID-19 typically present with fever, cough, and myalgia [3]. Digestive symptoms including diarrhea, nausea, and vomiting have been reported as clinical presentations of the patients infected with SARS-CoV-2 but were less common [3].

\section{Case Presentation}

\section{Patient Information}

A 56-year-old man with a history of hypertension, coronary artery disease required coronary artery bypass grafting, and ascending aortic aneurysm presented to our emergency department in Houston, Texas with a 2-day history of fever,

This article is part of the Topical Collection on COVID-19

Siraya Jaijakul

sjaijakul@houstonmethodist.org

1 Division of Infectious Diseases, Houston Methodist Hospital, Houston, TX, USA shortness of breath, nausea, vomiting, severe abdominal pain, and bloody bowel movements. Patient had not traveled outside Houston area, neither had he contacted with anyone known to have COVID-19.

\section{Clinical Findings}

Physical examination revealed temperature of $101.1^{\circ} \mathrm{F}$, blood pressure of 184/109 $\mathrm{mmHg}$, pulse of 143 beats per minute, respiratory rate of 16 breaths per minutes, and oxygen saturation of $98 \%$ on ambient air. Lung auscultation was clear. Abdominal examination revealed distended abdomen and generalized tenderness to palpation. Bright red blood was present on bed sheet.

\section{Diagnostic Assessment}

Blood tests on presentation (Table 1) revealed leukocytosis, lymphopenia, thrombocytopenia, elevated C-reactive protein, and lactic acid. Chest X-ray (CXR) showed bibasilar subsegmental atelectasis (Fig. 1). Computerized tomography (CT) scan of the chest, abdomen, and pelvic with contrast revealed no pulmonary airspace disease but demonstrated wall thickening of the ascending, transverse, and descending colon which was compatible with colitis (Fig. 2). Blood cultures, HIV serology, and acute viral hepatitis serology were negative. Stool gastrointestinal pathogen panel polymerase chain reaction (PCR) was negative. Given an ongoing COVID-19 outbreak in the community, nasopharyngeal swab was obtained for COVID-19 qualitative PCR test, which was reported as detected on the day of admission. 
Table 1 Laboratory findings during hospitalization

\begin{tabular}{|c|c|c|c|c|c|c|c|c|}
\hline Day & Day of admission & 1 & 2 & 3 & 4 & 5 & 6 & 7 \\
\hline \multicolumn{9}{|l|}{ Labs } \\
\hline WBC $(\mathrm{k} / \mu \mathrm{L})$ & 12.8 & 12 & 6.6 & 5.8 & 4.6 & 4.3 & 4.8 & 4.7 \\
\hline Neutrophils $($ cells $/ \mu \mathrm{L})$ & 11,264 & 9889 & 4745 & 3991 & 2742 & 2524 & 2405 & 2385 \\
\hline Lymphocytes (cells/ $\mu \mathrm{L})$ & 769 & 1184 & 1009 & 871 & 969 & 872 & 1120 & 1211 \\
\hline Hemoglobin (g/dL) & 16 & 15.8 & 12.2 & 13.9 & 11.2 & 11.1 & 12.1 & 10.5 \\
\hline Hematocrit (\%) & 44.8 & 43.8 & 36.5 & 40.5 & 32.8 & 33 & 35.6 & 31.9 \\
\hline Platelet $(\mathrm{k} / \mu \mathrm{L})$ & 133 & 105 & 89 & 114 & 136 & 157 & 204 & 233 \\
\hline \multicolumn{9}{|l|}{$\operatorname{ESR}(\mathrm{mm} / \mathrm{h}) 6$} \\
\hline CRP (mg/dL) & 8.52 & 21.47 & 24.95 & 15.19 & 11.45 & 7.37 & 5.91 & \\
\hline AST (U/L) & 67 & 38 & 24 & 25 & 30 & 32 & 35 & 29 \\
\hline ALT (U/L) & 82 & 54 & 35 & 33 & 29 & 31 & 37 & 31 \\
\hline LDH (U/L) & 265 & 196 & 141 & 171 & 149 & 140 & 154 & \\
\hline Lactic acid (mmol/L) & 2.9 & 1.6 & 1 & 1.5 & & & & \\
\hline Ferritin (ng/mL) & & 287 & & & 237 & & & \\
\hline Interleukin-6 (pg/mL) & 24 & & & & & & & \\
\hline Troponin (ng/mL) & $<0.006$ & 0.012 & & & & & & \\
\hline D-dimer $(\mu \mathrm{g} / \mathrm{mL})$ & 4.14 & & & & & & & \\
\hline COVID-19 PCR & Detected & & & & & & Not-detected & Not-detected \\
\hline
\end{tabular}

\section{Timeline and Therapeutic Intervention}

On hospital day 2, patient had persistent abdominal pain and bloody diarrhea with no respiratory symptom. His laboratory findings during hospitalization were showed in Table 1. His hemoglobin level dropped and he developed worsening thrombocytopenia and elevated CRP. Repeat CXR showed no pulmonary infiltrate. Enteral hydroxychloroquine $800 \mathrm{mg}$ once followed by $400 \mathrm{mg}$ daily in combination with ribavirin $400 \mathrm{mg}$ three times a day were initiated.

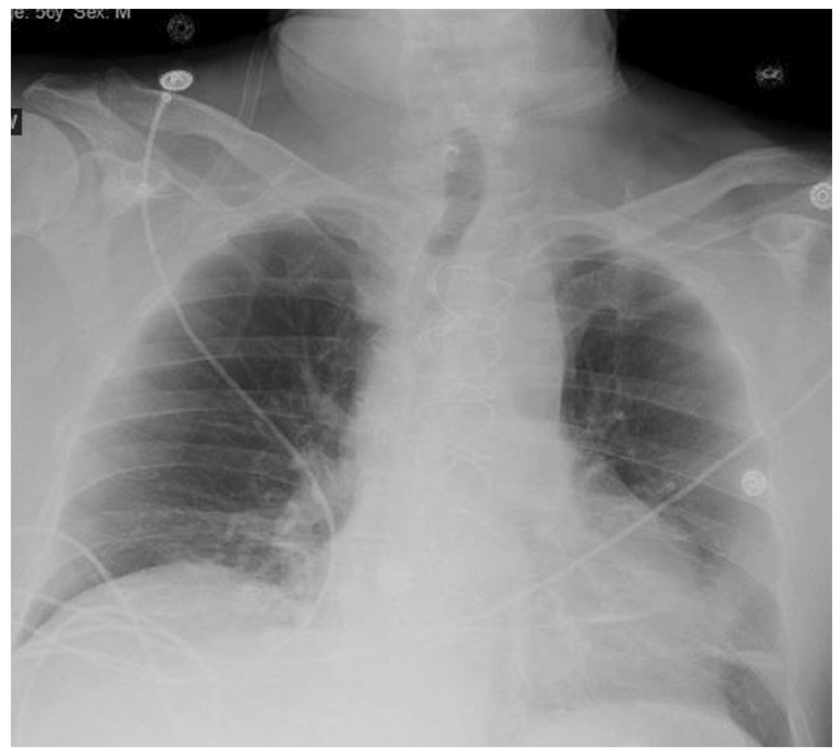

Fig. 1 Chest X-ray showed subsegmental bibasilar atelectasis

\section{Follow-Up and Outcomes}

His abdominal pain improved and bloody diarrhea stopped within the next $48 \mathrm{~h}$. His hemoglobin and hematocrit had been stable. Therefore, endoscopic examination was not performed during this admission. On hospital day 5, CT angiogram of abdomen and pelvic with intravenous contrast was performed

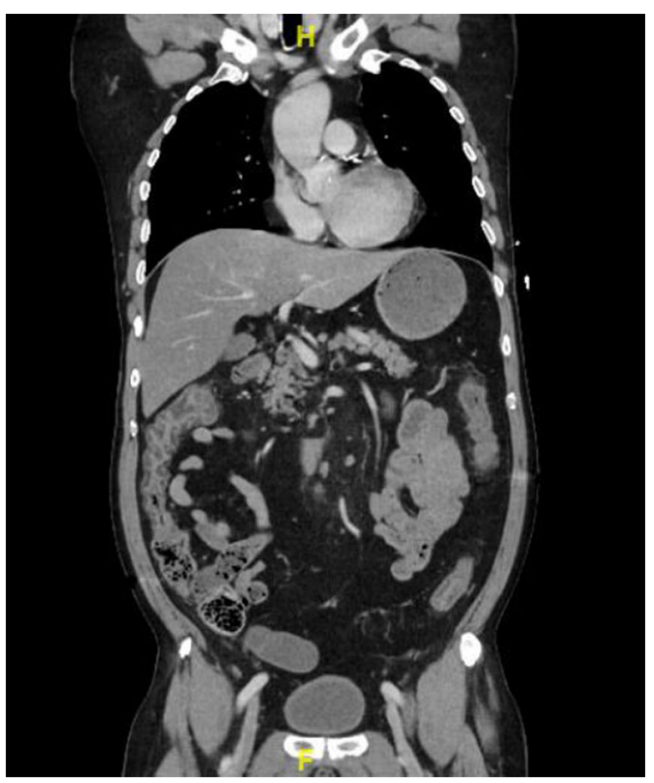

Fig. 2 CT abdomen and pelvic with contrast showed wall thickening of the ascending, transverse, and descending colon with mild pericolonic edema 
which did not reveal evidence of vascular abnormality but demonstrated improvement of colitis. His two sets of nasopharyngeal swabs collected for COVID-19 PCR were not detected on hospital day 6 and 7 consecutively. The patient was discharged on hospital day 7 after he completed a 5-day course of hydroxychloroquine and ribavirin with a satisfactory response in both clinical and laboratory findings.

\section{Discussion}

SARS-CoV-2 infection can uncommonly cause digestive symptoms [4] as the viruses bind to their target cells through angiotensin-converting enzyme 2 (ACE2) which are constitutively expressed by epithelial cells of the lung, kidney, blood vessels, and also intestine particularly terminal ileum and colon [5]. Fecal-oral route could potentially be another route of transmission [6] as SARS-CoV-2 viral nucleic acid was detected in stool of patients with COVID-19 [7, 8]. Sub-group of COVID-19 patients with mild disease could present with digestive symptoms alone and be more likely to test positive for COVID-19 PCR in the stool [9].

Bloody diarrhea was reported as a clinical manifestation of COVID-19 in a patient with underlying inflammatory bowel disease (IBD) [10]. Carvalho et al. has reported a case of SARSCoV-2 gastrointestinal infection in a patient without underlying IBD who initially presented with bloody diarrhea and subsequently developed pneumonia after hospitalization [11]. The patient was not promptly placed on SARS-CoV-2 isolation precautions and the diagnosis was not made until day 4 of hospitalization as the patient did not initially meet criteria for identifying persons under investigation (PUI) for SARS-CoV-2 infection by Centers for Disease Control and Prevention.

Limitations of our study are that the diagnosis was based on a clinical and laboratory findings as well as the clinical response of the patient. The diagnosis of gastrointestinal COVID-19 could be cumbersome in various institutes since stool study for SARS-CoV-2 PCR was not routinely performed or validated in their laboratory. Besides, endoscopic procedures are aerosol generating which could result in risk of COVID-19 transmission to healthcare personnel. Although histological examination of colon and rectal biopsies did not reveal any pathognomonic finding [11], the endoscopic examination would still be warranted to eliminate other potential etiologies.

To the best of our knowledge, this is the first report of COVID-19 patient presenting with colitis alone with no concomitant pneumonia. This report may promote physician awareness of extra-respiratory presentation of COVID-19 and emphasize clinical significance of more widely and rapidly testing SARS-CoV-2 PCR in fecal sample in patients presenting with gastrointestinal symptoms alone due to unexplained etiology during the COVID-19 pandemic.

\section{Compliance with Ethical Standards}

Conflict of Interest The author declares that she has no conflict of interest.

Ethics Approval and Consent to Participate Informed consent was unable to be obtained because the patient could not be traced after hospital discharge.

\section{References}

1. World Health Organization. WHO director-General's opening remarks at the media briefing on COVID-19-11 March 2020. Geneva: World Health Organization; 2020.

2. CDC COVID-19 Response Team. Preliminary estimates of the prevalence of selected underlying health conditions among patients with Coronavirus Disease 2019 — United States, February 12March 28, 2020. MMWR Weekly. 2020;69:382-6.

3. Guan WJ, Ni ZY, Hu Y, Liang WH, Ou CQ, He JX, et al. Clinical characteristics of coronavirus disease 2019 in China. N Engl J Med. 2020;382:1708-20. https://doi.org/10.1056/NEJMoa2002032.

4. Xiao F, Tang M, Zheng X, Liu Y, Li X, Shan H. Evidence for gastrointestinal infection of SARS-CoV-2. Gastroenterology. 2020;158:1831-1833.e3. https://doi.org/10.1053/j.gastro.2020.02. 055.

5. Harmer D, Gilbert M, Borman R, Clark KL. Quantitative mRNA expression profiling of ACE 2, a novel homologue of angiotensin converting enzyme. FEBS Lett. 2002;532:107-10.

6. Gu J, Han B, Wang J. COVID-19: gastrointestinal manifestations and potential fecal-oral transmission. Gastroenterology. 2020;158: 1518-9. https://doi.org/10.1053/j.gastro.2020.02.054.

7. Holshue ML, DeBolt C, Lindquist S, Lofy KH, Wiesman J, Bruce $\mathrm{H}$, et al. First case of 2019 novel coronavirus in the United States. N Engl J Med. 2020;382:929-36.

8. Wang W, Xu Y, Gao R, Lu R, Han K, Wu G, et al. Detection of SARS-CoV-2 in different types of clinical specimens. JAMA. 2020. https://doi.org/10.1001/jama.2020.3786.

9. Han C, Duan C, Zhang S, Spiegel B, Shi H, Wang W, et al. Digestive symptoms in COVID-19 patients with mild disease severity: clinical presentation, stool viral RNA testing, and outcomes [preprint]. Am J Gastroenterol. 2020;115:916-23 https://doi.org/ 10.14309/ajg.0000000000000664.

10. Mazza S, Sorce A, Peyvandi F, Vecchi M, Caprioli F. A fatal case of COVID-19 pneumonia occurring in a patient with severe acute ulcerative colitis. Gut. 2020;69:1148-9. https://doi.org/10.1136/ gutjnl-2020-321183.

11. Carvalho A, Alqusairi R, Adams A, Paul M, Kothari N, Peters S, et al. SARS-CoV-2 gastrointestinal infection causing hemorrhagic colitis: implications for detection and transmission of COVID-19 disease [preprint]. Am J Gastroenterol. 2020;115:942-6 https://doi. org/10.14309/ajg.0000000000000667.

Publisher's Note Springer Nature remains neutral with regard to jurisdictional claims in published maps and institutional affiliations. 